\title{
Delayed matching in pigeons with food and no-food samples: Further examination of backward associations
}

\author{
LOU M. SHERBURNE and THOMAS R. ZENTALL \\ University of Kentucky, Lexington, Kentucky
}

\begin{abstract}
When pigeons are trained on a delayed conditional discrimination with presence versus absence samples and tested with delays, a bias to choose the comparison associated with the absence sample is observed with increasing delay. Additionally, when the samples consist of food versus no food, this trial-type performance difference is reversed on short-delay trials: a bias to choose the comparison associated with the presence sample develops with delay testing. This reversal in comparison bias at short delays has been attributed to a preference produced by backward associations between the hedonic samples and the nonhedonic choice stimuli. In the present experiment, we tested an alternative hypothesis, that the short-delay comparison bias is produced by proactive interferencein particular, from reinforcement obtained on the previous trial-by including a group trained with reinforcement on only half of the trials with a correct response. According to the proactive interference account, this group should have shown a smaller short-delay comparison bias than would the typical $100 \%$ reinforcement group. Instead, consistent with a backward-association interpretation, the magnitude of the short-delay comparison bias shown by the $50 \%$ group was significantly greater than that shown by the $100 \%$ group.
\end{abstract}

Backward associative learning can be said to occur when the presentation of an unconditional stimulus (US) followed by a conditional stimulus (CS) results in a change in behavior to the CS (Zentall, Sherburne, \& Steirn, 1992).

According to Spetch, Wilkie, and Pinel (1981), the acceptance of backward associative learning (or conditioning) has been impeded by traditional views of Pavlovian conditioning processes (e.g., Hull, 1943; Rescorla \& Wagner, 1972). But a more important reason for the relative scarcity of reported research on backward conditioning is the difficulty in defining what the appropriate conditional response (CR) should be. When pairing occurs in the forward direction, the CR is often quite similar to the unconditioned response (UR). In the case of a CS-US association following backward US-CS pairing, however, it is not obvious what response would indicate the presence of such an association (Matzel, Held, \& Miller, 1988). Certainly one would not expect to observe a UR following the CS.

One approach to the study of backward conditioning has been proposed by Hearst (1989). Following repeated backward US-CS pairings, Hearst found facilitated acquisition of the forward CS-US association (relative to appropriate controls).

This research was supported by National Science Foundation Grants BNS-8418275 and BNS-9019080 and National Institute of Mental Health Grant 45979 to T.R.Z. The authors thank Janice N. Steirn and Karen L. Roper for their assistance with this research. Correspondence should be addressed to T. R. Zentall, Department of Psychology, University of Kentucky, Lexington, KY 40506.

\footnotetext{
-Accepted by previous editor, Vincent M. LoLordo
}

Another approach to the assessment of backward conditioning has been to examine the development of associations between two nonhedonic stimuli (i.e., two CSs). This approach has a long history of use in human verbal learning research (see, e.g., Asch \& Ebenholtz, 1962). For example, one can demonstrate that, after repeated S1-R1 pairings, human subjects will often respond with S1 when presented with R1.

The paired-associate procedures used with humans are analogous to conditional discrimination tasks used with animals. In conditional discrimination learning, pigeons, for example, are trained to respond to one comparison stimulus (C1) in the presence of one sample (S1) and to respond to the other comparison (C2) in the presence of the other sample (S2). One can then interchange samples and comparisons and ask about the development of backward $\mathrm{C} 1-\mathrm{S} 1$ and $\mathrm{C} 2-\mathrm{S} 2$ associations. When this procedure has been used, little evidence of backward associations has been found (Hogan \& Zentall, 1977; Richards, 1988). But, as noted by Zentall et al. (1992), in training, samples always appeared on the center response key, whereas comparisons always appeared on the side keys. Changing the location at which the stimuli appeared (as well as their temporal order) may have been sufficiently confusing to the pigeons to wash out evidence for backward conditioning.

Zentall et al. (1992) made use of a variation on the conditional discrimination task to demonstrate that forward associative training can result in the development of backward associations. The variation used was one in which correct responding following the presentation of one sample resulted in one outcome (i.e., food), whereas 
correct responding following the other sample resulted in a different outcome (i.e., an empty feeder). In this procedure, known as the differential outcomes procedure, pigeons are trained to associate correct responding to one comparison with food and correct responding to the other comparison with the empty feeder. On test trials, Zentall et al. replaced the visual samples presented on the center key with food on some trials and with the empty feeder on others. Test results indicated that there was significant control of comparison choice by the comparison-outcome associations learned in training. In other words, presentation of the food sample led to choice of the comparison that in training had been associated with food outcomes, while presentation of the empty-feeder sample led to choice of the other comparison.

Further evidence for the development of backward associations in pigeons using conditional discriminations was reported by Sherburne and Zentall (1993). In this study, retention functions were examined for samples that consisted of the presence versus the absence of food (i.e., food vs. an empty feeder). As had been reported in earlier studies in which food versus its absence had been used as samples, (see, e.g., Colwill, 1984; Grant, 1991; Wilson \& Boakes, 1985), the birds showed a bias to respond to the comparison associated with the absence sample on long-delay trials. Presence-sample-trial retention functions declined steeply with increasing delay between the samples and comparisons, whereas absencesample-trial retention functions remained high. This difference in the slope of retention functions has been interpreted as evidence that presence samples are "coded" and remembered, whereas absence samples are not. Instead, at the time of comparison choice, when the pigeons have no sample code, they respond to the alternative sample "by default" (see, e.g., Colwill, 1984; Grant, 1991; Wilson \& Boakes, 1985).

In addition to the long-delay comparison bias, Sherburne and Zentall (1993) also found that as delay testing progressed, a reversal of the bias could be observed on short-delay trials. On these trials, performance on foodsample trials was consistently more accurate than performance on no-food-sample trials. A similar short-delay bias to respond to the comparison associated with the food sample can also be observed in data presented by others (Colwill, 1984; Grant, 1991; Maki, 1979; Maki \& Hegvik, 1980; Wilson \& Boakes, 1985).

One interpretation offered by Sherburne and Zentall (1993) to explain the short-delay bias is that the longdelay bias to respond to the comparison associated with the absence sample results in a difference in the probability of reinforcement associated with responding to the two comparisons. Specifically, although responses to the absence-associated comparison are often made incorrectly after a long delay (i.e., when a presence sample is forgotten), responses to the presence-associated comparison (although they don't occur often) are rarely made incorrectly. Thus, the presence-associated comparison may come to be preferred because responding to it is reinforced more reliably.
Alternatively, Sherburne and Zentall (1993) suggested that the short-delay bias might emerge because of the development of backward associations between the hedonic samples and their associated comparisons. In other words, although correct responding to either of the comparisons resulted in a food outcome, the association of one of the comparisons with food samples led to a preference for that comparison. Experience with delays might encourage the formation of such comparisonsample associations by inducing sample rehearsal, thus increasing the likelihood that a representation of the food sample is available for association with the comparison response (see Wagner \& Terry, 1975).

Sherburne and Zentall (1993) tested the differential probability-of-reinforcement versus backward-association accounts by training pigeons with either hedonic presence/ absence samples (i.e., food vs. no food) or nonhedonic presence/absence samples (i.e., hue vs. no hue). They reasoned that, according to the differential probabilityof-reinforcement hypothesis, if the long-delay bias was observed in both groups, then the short-delay bias should also develop with delay testing in both groups. On the other hand, according to the backward-association interpretation, the short-delay bias should develop only in the group trained with hedonic samples, because only in this group should backward associations between comparisons and samples result in a preference for one of the comparisons (i.e., the one associated with the food sample). Consistent with the backward association account, although the long-delay bias was observed in both groups, the short-delay reversal in bias developed only in the group trained with hedonic samples.

An alternative account of the short-delay comparison bias found with food and no-food samples relies on proactive interference from the preceding trial(s). On delayed matching trials, there are two potential sources of interference: events occurring during the sample-comparison delay (retroactive interference) and those occurring prior to the trial (proactive interference). In a typical matching task, a specific source of proactive interference is the identity of the sample from the prior trial. But when food and no-food samples are used, there is an additional source of proactive interference, the outcome from the prior trial. Thus, when hedonic samples are used, one would expect interference not only from the prior sample but also from the prior outcome. Because performance on this task is typically well above chance, the last event on the prior trial is generally a food outcome. Thus, at the time of comparison choice, prior food presentations may interfere with memory for the sample, and a bias to respond to the food-sample-associated comparison may result. Although this food-outcome interference effect would be expected to be present at all delays, it is likely to be apparent only at short delays when other factors (e.g., asymmetrical coding effects) should have less of an effect on performance.

The purpose of the present study was to distinguish between the backward-association and the proactiveinterference accounts of the short-delay comparison bias 
found when pigeons perform food-versus no-food-sample delayed conditional discriminations. The strategy adopted here was to vary the proportion of correct responses reinforced between groups. If the short-delay bias is produced by proactive interference, reducing the probability of reinforcement following correct comparison responses should decrease the number of proactively interfering events and, thus, should reduce the magnitude of the bias. On the other hand, if the short-delay bias is produced by backward associations, reducing the probability of reinforcement might actually increase the strength of the backward association, or at least better allow evidence of the backward association to emerge. The reasoning is as follows: It is possible that evidence for a backward association is "overshadowed" by the strong forward association between the correct comparison and reinforcement. At a theoretical level, this overshadowing could occur if the capacity of a CS to support associations is limited and thus must be shared among USs paired with the CS both in the forward and backward directions. Therefore, it may be the case that the presence of a strong, forward, comparison-outcome association diminishes the potential for a strong, backward, comparison-sample association. Alternatively, the strong forward association may simply constrain the appearance of the weaker backward association. If reducing the probability of reinforcement following correct comparison responses weakens those associations and either allows for the formation of a stronger backward association or allows for evidence of the existing backward association to emerge, then (a) the bias to respond to the comparison associated with the food sample at short delays should be magnified, and (b) the bias should be evident at a longer delay relative to what has been found with $100 \%$ reinforcement of correct comparison responding.

\section{METHOD}

\section{Subjects}

The subjects were eight experimentally naive White Carneaux pigeons 5-8 years of age that were obtained from the Palmetto $\mathrm{Pi}$ geon Plant (Sumter, NC). The pigeons were housed in individual wire cages in a colony room which was on a $12: 12$-h on:off light cycle. The pigeons were maintained at $75 \%-80 \%$ of their freefeeding weights throughout the experiment, but they had free access to water and grit.

\section{Apparatus}

The experiment was conducted in a standard test chamber (BRS/LVE, Laurel, MD, Model 132-02) with inside measurements $33 \mathrm{~cm}$ high, $35 \mathrm{~cm}$ from front response panel to back wall, and $31 \mathrm{~cm}$ from side to side. The bottom edges of three horizontally aligned rectangular response keys $(3.0 \mathrm{~cm}$ wide, $2.5 \mathrm{~cm}$ high, and $0.8 \mathrm{~cm}$ apart) were located $21 \mathrm{~cm}$ above the floor. An in-line stimulus projector (Industrial Electronics Engineering, Van Nuys, CA, Model 10 with General Electric, No. 1820 lamps), mounted behind each response key, could illuminate the center key, white (W), and the side keys, red $(R)$ and green $(G)$. The $R$ and $G$ hues were produced by Kodak Wratten filters Nos. 26 and 60, respectively, and W was produced by the absence of a filter. Access to presentations of mixed grain (Purina Pro Grains) was permitted through a horizontally centered $5.2 \times 5.8 \mathrm{~cm}$ aperture, the bottom edge of which was $8 \mathrm{~cm}$ from the floor. The aperture was illuminated for $2 \mathrm{sec}$ whenever the feeder was raised. A shielded houselight (General Electric No. 1820), directed to the ceiling of the chamber, was mounted $4 \mathrm{~cm}$ from the top of the response panel. Extraneous sounds were masked by white noise at $72 \mathrm{~dB}$ presented through a speaker mounted behind the response panel and by noise from an exhaust fan mounted on the exterior of the chamber. Experimental contingencies were controlled by a microcomputer located in an adjacent room.

\section{Procedure}

Preliminary training. All the pigeons were trained to eat from the feeder and were then shaped by the method of successive approximations to peck $W$ on the center response key and $R$ and $G$ on each of the side keys. A single peck resulted in the presentation of $2 \mathrm{sec}$ of mixed grain (reinforcement). Pretraining continued until the birds responded to the center key 24 times and to each of the side keys 12 times ( 6 times to each hue presented on each key).

Conditional discrimination training. Conditional discrimination training began immediately following preliminary training. All conditional discrimination trials began with the onset of $W$ on the center key. A single response to the center key extinguished the key light and resulted in the 2 -sec presentation of mixed grain and the feeder light (food) or the feeder light alone (no food). $R$ and $G$ comparison stimuli then appeared on the side keys.

For half of the pigeons, responses to the $\mathrm{R}$ comparison were correct following samples of food and responses to the $\mathrm{G}$ comparison were correct following samples of no food. For the remaining pigeons, these contingencies were reversed. On all trials, a comparison response was followed by the offset of the comparison stimuli and a 10 -sec intertrial interval (ITI) with the houselight illuminated. The houselight was turned off at all other times. When an incorrect response was made, the ITI was followed by a repeat of the prior trial with the incorrect alternative omitted (i.e., a correction procedure). Only the first presentation of each trial was used in calculating performance. The number of each sample type, position of the correct comparison, and position of the hue comparisons was balanced across trials in each session. Two sequences of trial order were presented in double-alternating order. Each session consisted of 64 trials, and sessions were conducted 6 days a week.

The pigeons were assigned randomly to one of two groups. For pigeons in Group 100, all correct comparison responses resulted in 2-sec access to the feeder. For pigeons in Group 50, a random half of the correct comparison responses (with either type of sample) resulted in 2-sec access to the feeder, while the remaining correct comparison responses were followed by feeder light alone. Each pigeon was trained on the conditional discrimination to a criterion of $90 \%$ correct choices overall, on two consecutive sessions.

Delay testing. As each pigeon reached criterion on the conditional discrimination it was transferred to a mixed-delay procedure involving an equal number of trials with a $0-, 2-, 4-$, or $8-\mathrm{sec}$ delay between the offset of the sample and onset of the comparison stimuli. All stimuli were turned off during the delay. The correction procedure used in training was also used throughout the 10 sessions of delay testing.

\section{RESULTS}

\section{Acquisition}

In all analyses, the .05 level of significance was adopted. Pigeons in Group 100 acquired the conditional discrimination to criterion in an average of 19.0 sessions, whereas those in Group 50 acquired the task in an average of 11.8 sessions. A one-way analysis of variance (ANOVA) performed on the sessions to criterion scores indicated that this difference was not statistically significant $[F(1,6)=$ 
$5.06, p=.066]$. However, two of the birds in Group 100 showed a drop in performance after reaching $90 \%$ correct. When the analysis was performed again using a performance criterion of one session at $90 \%$ correct (rather than two), the difference was significant $[F(1,6)=$ 19.29]. Thus, it appears that Group 100 acquired the conditional discrimination more slowly than Group 50. Acquisition data for the first 10 sessions of training for the two groups appear in Figure 1.

As can be seen in Figure 1, superior performance by Group 50 is limited to later training sessions. In fact, early in training, Group 100 actually performed better than Group 50. This conclusion was confirmed by an analysis of sessions to a criterion of $60 \%$, which showed significantly faster attainment of criterion by Group 100 than by Group $50[F(1,6)=8.33]$.

A two-way ANOVA performed on performance scores for the first 10 training sessions for each group indicated a nonsignificant effect of group $[F(1,6)=3.71]$, a significant effect of sessions $[F(9,54)=50.00]$, and a significant group $\times$ sessions interaction $[F(9,54)=3.11]$.

For each of the two groups, within-subjects one-way ANOVAs were performed on food- versus no-food-sample trial performance, pooled over all training sessions. No trial-type performance difference (i.e., 0 -sec-delay bias) was evident in training in either group (both $F_{\mathrm{s}}<1$ ).

\section{Delay Test}

Delay performance, pooled over the 10 delay sessions, suggests that the retention functions on food-sample trials started at a higher level at 0 -sec delay and were steeper with increasing delays than were the retention functions on no-food-sample trials. Although overall delay performance by the two groups was comparable, the difference in 0-sec-delay performance on the two sample types was greater for Group 50 (33.2\% greater percent correct on food-sample trials) than for Group 100 (19.4\% greater percent correct on food-sample trials) and the superiority of food-sample trial performance ex-

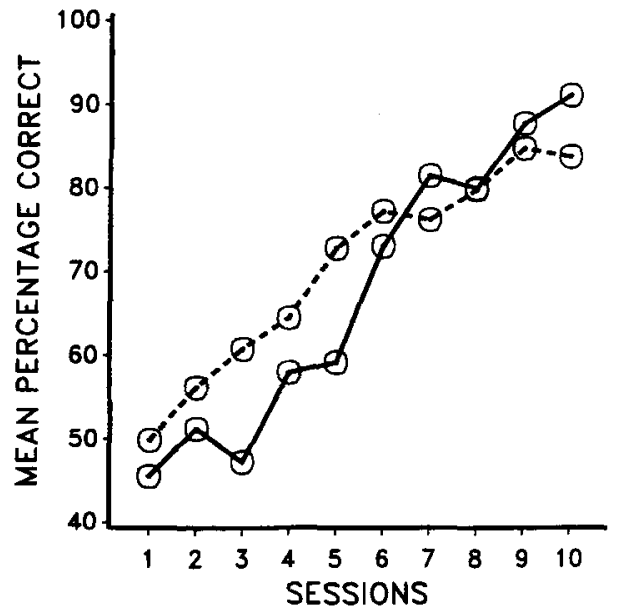

Figure 1. Acquisition of the food/no-food sample conditional discrimination by Group 50 (solid line) and Group 100 (dashed line).

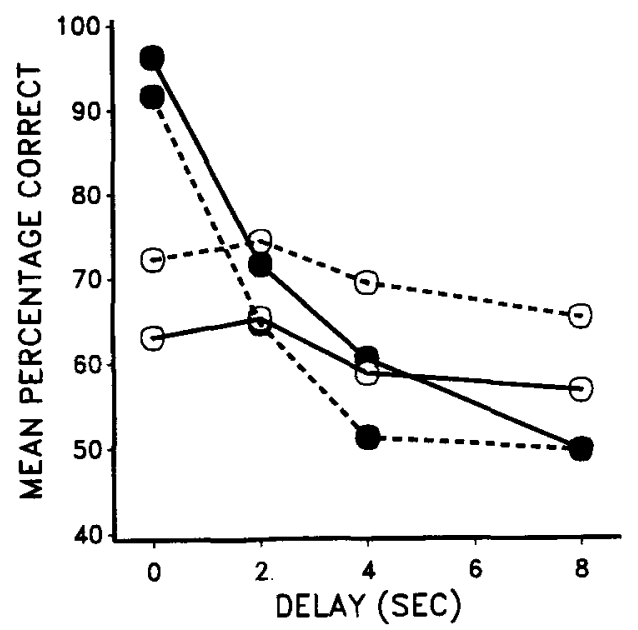

Figure 2. Delayed conditional discrimination performance for Group 50 (solid lines) and Group 100 (dashed lines) on food-sample trials (filled circles) and no-food-sample trials (open circles).

tended to a longer delay for Group 50 (between 4 and $8 \mathrm{sec}$ ) than for Group 100 (between 1 and $2 \mathrm{sec}$ ). The delay data appear in Figure 2.

A three-way ANOVA performed on the delay data, with group, sample type, and delay as factors, indicated that although neither the effect of group $(F<1)$ nor the effect of sample type $(F<1)$ was significant, the effect of delay was significant $[F(3,18)=59.38]$. Among the interactions, only the group $\times$ sample type $[F(3,18)=10.67]$ and the sample type $\times$ delay $[F(3,18)=36.81]$ were significant. For Group 50, overall performance was somewhat better on food-sample trials (69.5\% correct) than on no-foodsample trials $(61.0 \%$ correct), whereas for Group 100, the reverse was true $(64.3 \%$ and $70.4 \%$ correct, respectively). The sample type $\times$ delay interaction indicates that the slopes of the retention functions on food-sample trials were steeper than those on no-food-sample trials.

A separate two-way ANOVA performed on the 0-secdelay data indicated that although the effect of group was not significant $[F(1,6)=1.18]$, there were significant effects of sample type $[F(1,6)=324.25]$ and group $X$ sample type interaction $[F(1,6)=22.16]$.

\section{DISCUSSION}

The results of the present experiment confirm the asymmetrical food-sample versus no-food-sample delay functions reported in previous research (Colwill, 1984; Grant, 1991; Maki, 1979; Maki \& Hegvik, 1980; Sherburne \& Zentall, 1993; Wilson \& Boakes, 1985). They also confirm the short-delay bias to respond to the comparison associated with a food sample that has been previously observed in the context of delay testing and that has been interpreted as evidence for backward associations between the correct hue comparison and the hedonic sample (Sherburne \& Zentall, 1993).

Furthermore, the present research indicates that reducing the probability of reinforcement for correct re- 
sponding does not reduce the short-delay comparison bias, as would be predicted if the short-delay comparison bias resulted from proactive interference from the outcome experienced on the preceding trial. Instead, the magnitude of the bias actually increased. This result is consistent with the development of backward associations between samples and correct comparisons, if one assumes that a reduction in the probability of reinforcement weakens the forward associations between correct comparison and food outcome, and either allows for the development of a stronger backward association or simply allows more room for the existing backward association to appear.

One difference between the procedure used in the present experiment and those used in other demonstrations of excitatory backward conditioning involves the establishment of both forward and backward associations between CS and US. In this experiment, although responding to both comparisons was associated with food in a forward direction, responding to only one of the comparisons was associated with food in a backward direction. This variable, as well as that of experience with delays between US and CS, may be important in allowing for evidence of excitatory backward associations to emerge.

A curious finding in the present study was that although Group 100 acquired the conditional discrimination to a low criterion ( $60 \%$ correct) faster than Group 50, Group 50 reached a criterion of $90 \%$ correct faster than Group 100 . Although, as might be expected, $100 \%$ reinforcement appears to have facilitated initial acquisition, paradoxically, continuous reinforcement appears to have retarded attainment of high levels of performance. These results suggest that while continuous reinforcement may facilitate the acquisition of a conditional discrimination, it may not support high levels of terminal performance as readily.

\section{REFERENCES}

Asch, S., \& Ebenholtz, S. M. (1962). The principle of associative symmetry. Proceedings of the American Philosophical Society, 106, 135-163.
Colwill, R. M. (1984). Disruption of short-term memory for reinforcement by ambient illumination. Quarterly Journal of Experimental Psychology, 36B, 235-258.

GRANT, D. S. (1991). Symmetrical and asymmetrical coding of food and no-food samples in delayed matching in pigeons. Journal of Experimental Psychology: Animal Behavior Processes, 17, 186-193.

HEARST, E. (1989). Backward associations: Differential learning about stimuli that follow the presence versus the absence of food in pigeons. Animal Learning \& Behavior, 19, 280-290.

Hogan, D. E., \& ZentalL, T. R. (1977). Backward associations in the pigeon. American Journal of Psychology, 90, 3-15.

Hull, C. L. (1943). Principles of behavior. New York: AppletonCentury-Crofts.

MAKI, W. S. (1979). Pigeons' short-term memories for surprising vs. expected reinforcement and nonreinforcement. Animal Learning $\&$ Behavior, 7, 31-37.

MAKI, W. S., \& HEGVIK, D. K. (1980). Directed forgetting in pigeons. Animal Learning \& Behavior, 8, 567-574.

Matzel, L. D., Held, F. P., \& Miller, R. R. (1988). Information and expression of simultaneous and backward associations: Implications for contiguity theory. Learning \& Motivation, 19, 317-344.

ResCoRla, R. A., \& WAGNER, A. R. (1972). A theory of Pavlovian conditioning: Variations in the effectiveness of reinforcement and nonreinforcement. In A. H. Black \& W. F. Prokasy (Eds.), Classical conditioning (pp. 64-99). New York: Appleton-Century-Crofts.

RICHARDS, R. W. (1988). The question of bidirectional associations in pigeons' learning of conditional discrimination tasks. Bulletin of the Psychonomic Society, 26, 577-579.

Sherburne, L. M., \& Zentall, T. R. (1993). Coding of feature and no-feature events by pigeons in a delayed conditional discrimination. Animal Learning \& Behavior, 21, 92-100.

SPetCh, M. L., Wilkie, D. M., \& PineL, J. P. J. (1981). Backward conditioning: A reevaluation of the empirical evidence. Psychological Bulletin, 89, 163-175.

WAGner, A. R., \& Terry, W. S. (1975). Backward conditioning to a CS following an expected vs. a surprising UCS. Animal Learning \& Behavior, 3, 370-374.

Wilson, B., \& BOAKES, R. A. (1985). A comparison of the short-term memory performance of pigeons and jackdaws. Animal Learning \& Behavior, 13, 285-290.

Zentall, T. R., Sherburne, L. M., \& Steirn, J. N. (1992). Development of excitatory backward associations during the establishment of forward associations in a delayed conditional discrimination by pigeons. Animal Learning \& Behavior, 20, 199-206.

(Manuscript received January 5, 1994; revision accepted for publication July 4, 1994.) 